against any tentorial herniation as a cause of the oculomotor palsy.

Wilson et $a l^{1}$ recently described three patients with internal carotid artery occlusions who also had transient ocular motor palsies, generally recovering within hours. However, pupil dilatation and ptosis were observed for up to three weeks. The most likely mechanism was transient impairment of the nutrient circulation of the ocular motor nerves. For example, the oculomotor nerve receives its blood supply from an anastomotic plexus of small arteries which in turn is supplied from branches of the internal carotid artery within the cavernous sinus, from multiple branches of the maxillary artery, as well as from the ophthalmic artery anteriorly, and from the posterior cerebral and basilar arteries posteriorly. ${ }^{23}$ This rich blood supply from multiple sources could explain why oculomotor palsy is so unusual, even transiently, after carotid occlusions or carotid ligations for the treatment of aneurysms, ${ }^{4}$ since occlusion of several components of the nerve's nutrient supply is probably required to produce significant ischaemia. Thus in the oculomotor palsy in diabetes mellitus there are diffuse small vessel abnormalities in the vasa nervorum. ${ }^{3}$ Similarly, flow was absent in the ophthalmic and internal carotid arteries in our case and in those of Wilson et al. Whether the resulting oculomotor palsy is transient or permanent may perhaps depend on the adequacy of the collateral blood supply from the posterior circulation, or on the extent of segmental occlusion of the plexus of small vessels along the nerves themselves.

R KAPOOR B E KENDALI $M$ J G HARRISON Departments of Neurology and Radiology, The Middlesex Hospital,
London, UK

1 Wilson WB, Leavengood JM, Ringel SP, Bott AD. Transient ocular motor paresis associated with acute internal carotid artery occlusion. Ann Neurol 1989;25:286-90.

2 Parkinson D. A surgical approach to the cavernous portion of the carotid artery: anatomical studies and case report. J Neurosurg 1965; 23:474-83.

3 Asbury AK, Aldredge H, Harschberg R, Fisher $C M$. Oculomotor palsy in diabetes mellitus: a clinicopathological study. Brain 1970;93: 555-66.

4 McKissock W, Paine K, Walsh L. Further observations on subarachnoid haemorrhage. $J$ Neurol Neurosurg Psychiatry 1958;21:239-48.

Penicillamine treatment of Wilson's disease and optic neuropathy

We report a case of optic neuropathy associated with penicillamine treatment of Wilson's disease.

A twenty six year old woman presented with a one year history of progressive shaking of her hands and four months of shaking of her head. As a result she had had to give up her job on a production line. There was no family history of neurological or liver disease. She had a history of tachycardia and had taken disopyramide $100 \mathrm{mg}$ three times a day for three years. A mitral regurgitation murmur had been noted in the past.

On examination her pulse was $80 /$ minute and regular, and blood pressure was $115 / 60$. There was a mid and late systolic murmur loudest at the left sternal edge. Higher functions were intact, but her manner was disinhibited. Visual acuity was $6 / 9$ on the right and $6 / 6$ on the left. Fundoscopy showed normal optic discs. Slit lamp examination showed Kayser-Fleischer rings. The other cranial nerves were normal. She had titubation, tremor of the upper limbs, worse on the right and aggravated by movement, and cogwheel rigidity of both wrists. Reflexes, power and sensation were normal.

Serum copper was $7.0 \mathrm{micromol} / 1$ (Normal: 12-26 micromol/1), caeruloplasmin $70 \mathrm{mg} / 1$ (Normal: $190-450 \mathrm{mg} / \mathrm{l}$ ) and 24 hour urinary copper was $5 \cdot 1 \mathrm{micromol} / 24$ hours (Normal $<0.8 \mathrm{micromol} / 24$ hours). Biochemical screen, plasma glucose, and chest and skull radiographs were all normal. Haemoglobin was 119 to $136 \mathrm{~g} / \mathrm{l}$, white blood count 2.2 to $3.9 \times 10^{9} / 1$ with polymorphs 1.0 to $2.3 \times 10^{9} / 1$ and platelets 85 to $116 \times$ $10^{9} / 1$. The bone marrow was mildly hypocellular, with reduced numbers of erythroid and myeloid cells and megakaryocytes. A CT brain scan showed low densities in the thalami and cerebral peduncles. Echocardiography showed mild mitral valve prolapse.

A diagnosis of Wilson's disease was made and D-penicillamine $50 \mathrm{mg}$ three times a day was started.

Three weeks after starting penicillamine the patient presented with failing vision. A week after starting treatment she had developed a "red light" in the centre of both visual fields and then progressive blurring of vision. On examination both optic discs were pale and both pupils reacted sluggishly to light. Corrected visual acuity was $6 / 24$ on the right and $6 / 18$ on the left. Near vision was N18 bilaterally. Visual evoked potentials (VEP) showed latencies of $102 \mathrm{~ms}$ on the right and $112 \mathrm{~ms}$ on the left (Normal $<115 \mathrm{~ms}$ ). Brainstem auditory (BAEP) and somatosensory evoked potentials (SSEP) were bilaterally delayed. The wave form of the BAEPs was small. Electroretinogram and autoimmune profile were normal.

Penicillamine was stopped and pyridoxine $50 \mathrm{mg}$ twice a day was started. Nine days later near vision was N10 in both eyes. Trientene dihydrochloride $600 \mathrm{mg}$ three times a day was started. Three weeks after stopping penicillamine colour vision had returned to normal. A week later pupillary reactions were improved and near vision was N6 (right) and N4 (left). The VEPs showed improved amplitude, but the latencies were unchanged. Three months after stopping penicillamine the VEP latency on the left had improved to $99 \mathrm{~ms}$ and on the right was $106 \mathrm{~ms}$. The BAEPs and SSEPs were unchanged. After seven months the visual acuity was $6 / 12$ on the right and $6 / 9$ on the left. After a year of trientene the tremor had greatly improved. She has since returned to work and her manner appears normal.

No case of untreated Wilson's disease with optic neuropathy has been described. BAEPs are commonly delayed in Wilson's disease, but delayed VEPs have been found in only a minority of cases with neurological involvement. ${ }^{1}$ Recovery of VEP latency with treatment has been shown in one study. The abnormal VEPs may be related to cerebral hemisphere involvement. ${ }^{1}$

Optic neuropathy in Wilson's disease treated with DL-penicillamine and D-penicillamine has been attributed to penicillamine induced pyridoxine deficiency: in two cases the optic neuropathy developed only after months at higher doses of penicillamine and improved with pyridoxine. Against this theory is a third case, ${ }^{2}$ which developed while on prophylactic pyridoxine. In all cases there was an improvement of the Wilson's disease with penicillamine treatment, making the Wilson's disease itself unlikely to be the cause of the optic neuropathy.

Optic neuropathy has been described in association with $\mathrm{D}$-penicillamine treatment of chronic active hepatitis for two months and rheumatoid arthritis for six months and for year. The last was associated with development of antinuclear antibody titre of $1 / 320$ and improved with steroids. ${ }^{3}$

This case differs from the others described as the duration of treatment before the development of optic neuropathy is much shorter. Pyridoxine deficiency is unlikely, because of the low dose and short duration of treatment. The short history and negative autoimmune profile make autoimmune disease unlikely. Neurological deterioration in the first month of penicillamine treatment of Wilson's disease has been recognised recently ${ }^{4}$ and the time course in this case would be consistent although optic neuropathy has not been described in this situation. Worsening of extrapyramidal signs is often seen in this syndrome, but was absent in this case. The neurological deterioration may be due to redistribution of copper. Trientene therapy has not been associated with neurological deterioration. Neuro-ophthalmic complications of desferrioxamine, including one case with optic neuropathy, have been associated with raised cerebrospinal fluid copper levels and attributed to redistribution of copper. Another explanation of the optic neuropathy in this case consistent with the short history would be an idiosyncratic hypersensitivity reaction. Although optic neuropathy is a rare complication, physicians should regularly assess the visual acuity of their patients on penicillamine, particularly when starting treatment or increasing the dose.

AHS LEE NF LAWTON Wessex Neurological Centre Southampton General Hospital, Southampton, UK

Correspondence to:

Dr Lee, Histopathology Department, Level E South Block; Southampton General Hospital, Southampton SO9 4XY, UK

1 Chu N-S. Sensory evoked potentials in Wilson's disease. Brain 1986;109:491-507.

2 Goldstein NP, Hollenhorst RW, Randall RV, Gross JB. Possible relationship of optic neuritis, Wilson's disease and DLpenicillamine therapy. JAMA 1966;196: 734-5.

3 Klingele TG, Burde RM. Optic neurowathy with penicillamine therapy in a patient with rheumatoid arthritis.
ophthalmol 1984;4:75-8.

4 Starosta-Rubinstein S, Young AB, Kluin K, et al. Clinical assessment of 31 patients with Wilson's disease. Correlations with structura changes on magnetic resonance imaging. Arch Neurol 1987;44:365-70.

5 Pall HS, Williams AC, Blake DR. Deterioration of Wilson's disease following the start of penicillamine therapy. Arch Neurol 1989;46:359-60.

\section{Serum erythropoietin levels in von Hippel-Lindau syndrome}

No serum marker exists in von HippelLindau syndrome (HLS), an autosomaldominant inherited cancer-prone disorder 